\title{
Research progress on the carcinogenesis mechanism of inflammation in ulcerative colitis: a narrative review
}

\author{
Peng Tang ${ }^{1 \#}$, Lei Zha ${ }^{2 \#}$, Chun Ye $^{3}$, Lihua Zhou ${ }^{4}$ \\ ${ }^{1}$ Department of Gastroenterology, Eastern Hospital, Sichuan Academy of Medical Sciences and Sichuan Provincial People's Hospital, University of \\ Electronic Science and Technology of China, Chengdu, China; ${ }^{2}$ Intensive Care Unit, Sichuan Academy of Medical Sciences \& Sichuan Provincial \\ People's Hospital, University of Electronic Science and Technology of China, Chengdu, China; ${ }^{3}$ State owned Assets Management Office, Sichuan \\ Academy of Medical Sciences \& Sichuan Provincial People's Hospital, University of Electronic Science and Technology of China, Chengdu, China; \\ ${ }^{4}$ Digestive Endoscopy Center, Sichuan Academy of Medical Sciences \& Sichuan Provincial People's Hospital, University of Electronic Science and \\ Technology of China, Chengdu, China \\ Contributions: (I) Conception and design: P Tang, L Zha; (II) Administrative support: P Tang, L Zhou; (III) Provision of study materials or patients: C \\ Ye, L Zhou; (IV) Collection and assembly of data: None; (V) Data analysis and interpretation: None; (VI) Manuscript writing: All authors; (VII) Final \\ approval of manuscript: All authors. \\ "These authors contributed equally to this work and should be considered as co-first authors. \\ Correspondence to: Chun Ye; Lihua Zhou. Sichuan Academy of Medical Sciences \& Sichuan Provincial People's Hospital, University of Electronic \\ Science and Technology of China, 32 w. Sec 2, 1st Ring Road, Qingyang District, Chengdu 610072, China. \\ Email: cyechun@163.com; zlh0828342@163.com.
}

\begin{abstract}
Objective: In this review, we have summarized the influence of inflammation-related pathological mechanisms on the development of ulcerative colitis (UC) to colorectal cancer (CRC).

Background: UC is a chronic inflammatory bowel disease (IBD) of unknown etiology that affects the colon and rectum. Long-term inflammation of UC may greatly increase the risk of CRC, and the secretion of inflammatory factors and sustained inflammation may be key drivers of UC-associated CRC progression. Compared with the general population, the risk of CRC in patients with UC is 2.4 times higher, and the mortality rate of patients with UC is higher than that of those with sporadic CRC. The use of non-steroidal anti-inflammatory drugs can reduce the probability of UC transforming into CRC.

Methods: Literatures about inflammation and UC were extensively reviewed to analyze and discuss.

Conclusions: We believe that the mechanism of continuous inflammation that promotes cancer in UC may be the result of the mutual influence of intestinal microbes, inflammatory signals, and tissue remodeling. The invasion of intestinal microorganisms activates inflammatory signals and promotes the secretion of inflammatory factors which intensifies the remodeling of the extracellular matrix (ECM) and recruits immune cells. Eventually, a mutually engendering circuit of microbial invasion, release of inflammatory mediators, and remodeling of ECM is formed, which triggers continuous inflammation and promotes development of CRC.
\end{abstract}

Keywords: Ulcerative colitis (UC); Crohn's disease (CD); gut microbes; inflammatory signals

Submitted Sep 26, 2021. Accepted for publication Nov 16, 2021.

doi: $10.21037 /$ apm-21-3138

View this article at: https://dx.doi.org/10.21037/apm-21-3138

\section{Introduction}

Ulcerative colitis (UC) is a common chronic inflammatory bowel disease (IBD) which happens in part or all of the mucosa and submucosa of the colon. It is characterized by urgent bowel movements, tenesmus, bloody diarrhea, abdominal pain, and fatigue (1). In the past 20 years, with the rapid industrialization in the south and east of China, the incidence of UC in these areas has risen swiftly. The 
incidence of UC in these industrialized areas is close to that of developed countries (2). The exact pathogenesis of UC is still unknown, although currently the main influencing factors are understood to include immune response disorders, changes in intestinal microbiota, genetic susceptibility, and environmental factors (3). In spite of the growing efforts of researchers in response to the increasing incidence of $\mathrm{UC}$, its management is still limited. According to the guidelines of the American Gastroenterology Association (AGA), in addition to surgical treatment when necessary, corticosteroids should be used to prevent inflammation in the acute phase. Similarly, longterm treatments are mainly steroidal and non-steroidal antiinflammatory drugs and immunomodulators $(4,5)$. These drugs often have a wide range of side effects, and some remain controversial.

Along with the suffering caused by UC, the long-lasting inflammation in UC could possibly greatly increase the risk of colorectal cancer (CRC). Overall, the incidence of CRC in patients with $\mathrm{UC}$ is about 2.4-fold higher than people without UC (6). Perhaps UC patients themselves are prone to $\mathrm{CRC}$-inducing genetic mutations. Some researchers have estimated that the incidence of CRC in UC patients after 30 years of UC diagnosis could be as high as $20 \%$ or more (7). Considering this bias, the incidence of CRC in UC patients has modestly decreased, especially in developed countries (6). However, as the incidence of $\mathrm{UC}$ has been increasing significantly with the process of social industrialization (2), the number of CRC cases potentially induced by $\mathrm{UC}$ is increasing rapidly, especially in developing countries like China.

The secretion of inflammatory factors and the persistence of inflammation may be key promoting forces in the progression of UC-related CRC, including in its occurrence, development, and progression. Intestinal microbial infection, inflammatory signaling activation, inflammatory cell infiltration, and inflammatory cytokine release in intestinal epithelial cells could all support the CRC promoting inflammation. The CRC promoting inflammation could further lead to chronic inflammation, inflammatory hyperplasia, abnormal hyperplasia, and tumor development (8). For example, in patients diagnosed with UC for the first time, interleukin (IL)-17A, a typical inflammatory cytokine, can predict the severity of the disease very well, and also the course of the disease in the future 3 years (9). It may also be involved in the occurrence and development of CRC, as it was shown to be more highly expressed in CRC than in UC, and rarely detected in normal tissues (10). Anti-inflammatory therapeutics such as antiIL-17A can significantly alleviate inflammation in UC and reduce the risk of CRC in UC mice model (11). Exploring the mechanism of inflammation in the carcinogenesis of UC may help prevent and treat UC-related CRC. In this review, we have summarized the current research progress in the pathogenesis of CRC carcinogenesis with respect to infection, tissue remodeling, inflammatory cytokine secretion, and inflammation activation in UC. We propose that mutual influence of intestinal microbes, inflammatory signals, and tissue remodeling all contribute to the continuous inflammation that promotes UC-related CRC. We present the following article in accordance with the Narrative Review reporting checklist (available at https:// dx.doi.org/10.21037/apm-21-3138).

\section{CRC and gut microbiota}

Including both UC and Crohn's disease (CD), IBD is considered an abnormal mucosal immune response of the body to the gut microbiota under the influence of environmental factors, pathogens, and genetic susceptibility (12). Under physiological conditions, the intestinal barrier is composed of epithelial cells, extracellular matrix (ECM) components, and a mucus layer with a complex physical and chemical structure. The barrier could protect the intestine from infection and unnecessary attack of the innate and the adaptive immune system. When UC occurs, the intestinal mucosal barrier is attenuated, which allows intestinal microorganisms to invade into the mucosa (13). In more than $80 \%$ of the colon specimens of UC patients, intestinal bacteria were found in the lesions where the mucosa was destroyed (14). In mouse models of colitis induced by azomethane (AOM) or dextran sodium sulfate (DSS), intestinal microorganisms were detected in the mucosal layer much earlier than leukocyte infiltration or changes in epithelial structure (15). These findings indicate that the invasion of intestinal microorganisms may be an important driving force for the occurrence of UC.

Gut microbes are made up of a large number of different bacteria that produce a variety of metabolites. A wellestablished feature of CRC development is that most cancers originate as precursors to benign polyps (16). In the majority of cases, CRC develops from adenomatous polyps through dysplastic alterations that are strongly influenced by the microbiota (17). It is estimated that at least $0.2 \%$ to $8.3 \%$ of colorectal polyps are malignant (18). A variety of molecular changes may occur during the 
transformation of these polyps from benign to malignant tumors, so the ability to study metabolites produced by the microbiome could lead to new discoveries about the development and progression of this cancer. Patients with $\mathrm{UC}$ are prone to colorectal cancer and dysplastic polyps, as well as sporadic adenomas (7). Previous studies have assessed the incidence of dysplastic lesions in UC patients and found that the incidence of adenomatous polyps in IBD is extremely low, only about $2 \%(19,20)$. This may be due to the beneficial protection of chemical drugs in $\mathrm{UC}$ and the inhibition of the inflammatory process of UC in adenoma development (21). Although a low incidence of dysplastic polyps is observed in patients with UC, this should not preclude close monitoring of this high-risk population.

Gut microbes may also play an important role in the development of UC-related CRC. There is an interaction between the intestinal flora and monocytelike macrophages. The typical interactive effect is the activation of the Toll-like receptor (TLR) of immune cells with bacteria-derived lipopolysaccharide (LPS). Activation of TLR mediates the secretion of inflammatory cytokines such as IL-1 $\beta$ and IL-17, which in turn activates T cell helper cells and the corresponding cancer-promoting inflammatory response (22). Intestinal microbes can also affect the inflammation and tumor transformation of cells with their metabolites. There is a reduced diversity of gut microbes in each single patient with IBD. The influence of disease processes, alterations of disease on the microbiome, and differences in the diet and drug use of patients all possibly bring about big differences in the microbial metabolome between different UC patients. Metabolites of the microbes can be used as a fine indicator of the disease course. Conversely, specific metabolites from UC patients, such as fecal calprotectin (FC), can also be used as a biomarker in diagnosing UC (23). In patients with $\mathrm{UC}$ who received microbiota transplantation, changes in bacterial species and metabolites were also observed as the condition improved (24). Among the many metabolites, linoleic acid and 12-hydroxy-8,10-octadecadienoic acid were implicated to have strong correlations with the development of UC-related CRC, suggesting that these metabolites can be used as key biomarkers for the development of UC-related CRC (25). The composition and metabolism of gut microbes are the decisive factors in the occurrence of UC-related CRC. However, whether either the gut microbes or their metabolites should be responsible for the inflammatory and carcinogenesis effects of UC is still needs further research (23).

\section{TLR4/nuclear factor kappa B pathway}

Microorganisms activate inflammatory lesions in UC. The glycolipid LPS is widely found in the cell walls of Gramnegative bacteria. It targets TLR of epithelial cells under infection, which further activates downstream inflammatory pathways such as nuclear factor kappa B (NF-кB) (26). As the most important inflammation-controlling hub that regulates the transcription of cytokines, cytokine receptors, and adhesion molecules, NF- $\kappa B$ participates in the regulation of multiple pathological processes of UC and CRC, including proliferation, apoptosis, metastasis, and drug resistance (27). Inhibitor of $N F-\kappa B$ kinase subunit beta $(\mathrm{IKK} \beta)$ is a key regulator of NF- $\kappa B$ activation. In the AOM/DSS-induced UC mouse model, the IKK $\beta$ knockout mice were less likely to develop CRC. Similarly, compared with healthy controls, in the bone marrow-specific IKK $\beta$ knockout mice, tumorigenesis was inhibited, and the pro-inflammatory cytokines in the intestinal mucosa such as IL-1 $\beta$, ICAM, IL-6, MIP-2, KC, COX-2, and matrix metalloproteinases (MMP)-9 were significantly reduced (28). To summarize, both the UC lesions and immune cells could be activated by classical and non-classical NF- $\mathrm{KB}$ signals to promote the inflammation and progression of UC.

In multiple cancers, including $\mathrm{CRC}, \mathrm{NF}-\kappa \mathrm{B}$ has been characterized as a tumor promoting regulator. Recent studies have shown that a variety of potential CRC suppressive chemicals, such as curcumin, dexamethasone, resveratrol, evodia, and so on, can inhibit NF- $\mathrm{KB}$ directly or indirectly. The inhibition of NF- $\mathrm{KB}$ with these chemicals could suppress tumor proliferation, inflammation, metastasis, and drug resistance, and could promote its apoptosis (27). As $\mathrm{NF}-\kappa \mathrm{B}$ interacts extensively with other cancerpromoting processes and molecules, it can regulate tumor progression and metastasis by promoting the induction of angiogenesis-related genes (29) (e.g., S100B, a common inflammatory protein in tumors). The levels of S100B in healthy control, peritumoral, UC, and CRC tissues increased with aggravation of the lesion. By directly binding to wtp53 and phosphorylating NF- $\kappa \mathrm{B}, \mathrm{S} 100 \mathrm{~B}$ up-regulates pro-inflammatory inducible nitric oxide synthase (iNOS), vascular endothelial growth factor (VEGF), and IL-6, inhibits apoptosis-inducing wtp53 and Bax, and forms a proinflammatory/angiogenic and anti-apoptotic environment. Inhibition of S100B has been shown to inactivate NF- $\mathrm{KB}$ and reduce CRC incidence (30). Other NF- $\mathrm{BB}$ interacting molecules mainly include IL-6/STAT3 (31), Notch, MMP, Cyclin, survivin, Fas, interleukin, TNF, and so on (27). 
There have been many studies on NF- $\kappa \mathrm{B}$ and tumors, but currently there is no effective and specific anti-tumor therapy that targets NF- $\mathrm{KB}$ in clinical practice. As a disease characterized by persistent inflammation, UC may be more likely to benefit from therapies such as anti-NF- $\kappa \mathrm{B}$ in reducing the incidence of CRC.

\section{Tumor necrosis factor- $\alpha$}

The tumor necrosis factor (TNF)- $\alpha$ protein is a main inflammatory mediator and is mainly expressed in infiltrating macrophages in UC $(8,32)$. Immunoreactive TNF- $\alpha$ protein can be detected in the colon of patients with active UC and advanced CRC, but not in normal mucosa; similar effects are also seen in AOM/DSS-induced UC mouse models (33). Both the TNF- $\alpha$ chimeric monoclonal antibody $(\mathrm{mAb})$ and inhibitor can significantly limit the level of inflammation in the colon and reduce the number and size of tumors in AOM/DSS challenged mice $(33,34)$. Clinically, TNF- $\alpha \mathrm{mAb}$ has a good effect on IBD patients, including those with UC and CD (35), which further illustrates the importance of TNF- $\alpha$ to $\mathrm{CD}$.

The expression of TNF receptor (R2) is also upregulated in IBD patients and AOM/DSS challenged mice $(34,36)$. However, the up-regulated subtype TNFR2 cannot not activate the $\mathrm{FADD} /$ caspase 8/caspase 3 pathway to promote apoptosis. Simultaneously, the activation of inflammation and proliferation with TNFR2 is enhanced (37). Furthermore, TNFR2 could activate NF- $\kappa \mathrm{B}$ and other inflammatory pathways to form a pro-inflammatory positive feedback (34). In recent years, it has been discovered that the expression of metastasis-associated in colon cancer 1 (MACC1) gene may be initiated by the activation of $\mathrm{TNF}-\alpha$ dependent NF$\kappa \mathrm{B}$ p65, which promotes the activation of the transcription factor c-Jun. These interactions may jointly promote the occurrence and development of UC-related CRC (8).

\section{IL-6}

Although the role of TNF- $\alpha$ in the development of UCrelated CRC has been widely accepted, TNF- $\alpha^{-/-}$mice have been found to still develop UC and related CRC with a similar severity to normal mice under the stimulation of AOM/DSS (38). There are multiple ways of inflammation activation, which could all play important roles in the progression of UC and related CRC. Similar to TNF- $\alpha$, IL-6 is also a typical multifunctional NF- $\kappa \mathrm{B}$ regulatory cytokine. The circulating and intestinal IL-6 levels of patients with IBD are elevated, and the level of IL-6 in the lesion is higher than that in normal tissues $(39,40)$. In the AOM/DSS mice model, IL-6 expression also increased with the aggravation of the lesion. Specifically, the number of tumors in IL- $6^{-1-}$ mice decreased, and the size of tumors were smaller $(41,42)$. These studies indicated that IL-6 may be a key promoter in the development of $\mathrm{UC}$ and related CRC.

After IL-6 binds to sIL-6R, its receptor of soluble form, it mediates chronic inflammation both in UC and CRC through trans-signal transduction (43). In epithelial cells of mucosa associated with UC and CRC, IL-6R messenger RNA (mRNA) is down-regulated, while the expression of secreted sIL-6R is increased, which would promote tumorigenesis of CRC (44). Other targets of IL-6 include JAK/STAT3, Shp-2-Ras, PI3K/AKT, and so on. Interactions between IL- 6 and these targets can further activate other inflammatory factors such as TNF- $\alpha$. In short, IL-6 may mainly bind to its soluble form receptor sIL-6R to promote the occurrence and development of UC and CRC.

\section{Other major CRC promoting inflammatory signals}

\section{The PGE2/COX-2 patbway}

Cyclooxygenase (COX) mainly includes COX-1 and COX-2, and its signal transduction is related to tumorigenesis (45). The expression of COX-2 only happens in response to certain pro-inflammatory stimuli. Pro-inflammatory cytokines induce the expression of COX2 and PGE2 and participate in vasodilation, leading to inflammation. It is also involved in inducing the expression of BCL-2, inhibiting apoptosis, and promoting tumor progression (46). In human CRC endothelial cells, PGE2 directly enhances the expression of CXCL1, a chemokine that can activate and recruit neutrophils and induce angiogenesis (47).

\section{The IL-23/Th17 pathway}

Dendritic cells and other antigen-presenting cells produce IL-23, a member of the IL-12 cytokine family. Since IL-23 receptors (IL23R) could regulate the differentiation of helper T cells (Th17), they are widely associated with chronic inflammatory diseases (26). Ustekinumab, an IL-12 and IL-23 monoclonal antibody, can be used for psoriasis, psoriatic arthritis, and CD, and also shows a certain clinical effect on UC $(48,49)$. 


\section{ECM}

In UC, series of aggressive metabolites and inflammatory mediators accumulate in the inflamed intestinal mucosa, leading to tissue damage (50). Tissue damage repair, especially immune response-enabled ECM degradation and remodeling, may be one of the most important pathologies of UC (51). On the one hand, ulcers in the pathological manifestations of UC itself tell a story of tissue damage repair, especially ECM damage and remodeling. On the other hand, the secretion of aggressive metabolites and inflammatory mediators is activated in UC, including nitric oxide, prostaglandins, oxygen free radicals, histamine, MMPs, and other proteases. These aggressive metabolites and inflammatory mediators may be the natural responsive products of the immune system to infection and trauma. However, the metabolites and inflammatory mediators could also aggravate tissue damage, perturb ECM remodeling, and perpetuate the disease in $\mathrm{UC}$ (52). The disorder of ECM remodeling in UC may be an important factor leading to progression of UC and UC-related CRC. For example, MMPs, under physiological conditions, are mainly involved in embryogenesis and angiogenesis, allowing inflammatory cells to migrate to the damaged sites of the tissue. Under pathological conditions, these MMPmediated migration processes may also induce ulcer healing disorders, sustained inflammation, and even cancer (53). Under continuous inflammatory signals such as the invasion of intestinal bacteria on the lesion and the secretion of inflammatory factors in tissues, the transcription, translation, secretion, and activation of MMPs are enhanced, basement membrane and ECM are hydrolyzed, and chemokines are secreted $(52,54)$. Such a process may form a continuous positive feedback loop that drives UC.

The content and way of combination of ECM components also have a great influence on local inflammation in UC. For example, the ECM component hyaluronic acid hardly adheres to white blood cells under normal conditions. When inflammation occurs, hyaluronic acid forms a complex with trypsin inter- $\alpha$ inhibitor, which attracts a large amount of white blood cells to activate inflammation (55). A similar mechanism is also found in the early stage of IBD tissue, where change in content and way of combination of ECM components happens to enable leukocyte infiltration, adhesion, and activation (56). Other ECM components including laminin, heparan sulfate proteoglycan, fibronectin, keratin, and dermatan sulfate have widely been reported as closely related to the occurrence and development of
UC (57). The TGF- $\beta$ protein is often considered the main regulatory protein of ECM remodeling. Recent studies have asserted that simply inhibiting the expression of TGF- $\beta$ in epithelial cells in the inflammatory microenvironment may lead to the occurrence of aggressive CRC (58). In colonic epithelial component-specific MMP-9 transgenic homozygous mice, reactive oxygen species accumulated and DNA damage reduced, which inhibited the occurrence and development of CRC (59). Tripartite motif-containing 21 (TRIM21) is a protein that regulates the immune response of the intestinal mucosa. The expression of TRIM21 is reduced in CRC patients. In the TRIM21 highly expressed mouse model, the tumor cell proliferation-related gene Ki67; the tissue remodeling and angiogenesis-related genes MMP10, HIF1- $\alpha$, COX2, Ang4; and the pro-inflammatory cytokines IL-6, TNF- $\alpha$, and IL- $1 \beta$ are all significantly up-regulated. While TRIM2 1 expression leads to down-regulation of the tumor cell adhesion-related gene E-cadherin and inflammatory cytokines IL-10, TGF- $\beta$, and Foxp3, which inhibits tumor occurrence and development (60). All these findings indicate that disordered ECM and its interaction with inflammatory signals may be effective targets in the treatment of CRC. In short, ECM changes or remodeling participate in many aspects of UC. However, the importance of ECM in the development of UC and related CRC has not attracted enough attention, much about its role in the development of UC-related CRC requires further investigation.

\section{Summary}

For the prevention of the UC-related CRC, timely control of the disease, regular screening, and healing of the damaged mucosa as soon as possible are the best strategies (6). Besides treatment when in the acute phase, often requires sustained use of non-steroidal anti-inflammatory drugs to control chronic inflammation, otherwise uncontrolled chronic mucositis may become a potent driving force for the development of UC and related CRC.

A variety of factors including intestinal microbes, inflammatory mediators such as NF- $\kappa \mathrm{B}$, TNF- $\alpha$, IL-6, PEG2, and so on, and the remodeling of ECM all have direct or indirect effects on the pathology and inflammation of UC. Generally, the remodeling of ECM makes it easier for microorganisms to penetrate the mucus layer and invade epithelial cells. The invasion of intestinal microbes activates TLR4 and other inflammatory signal transduction in the body to promote the secretion of inflammatory factors, and 


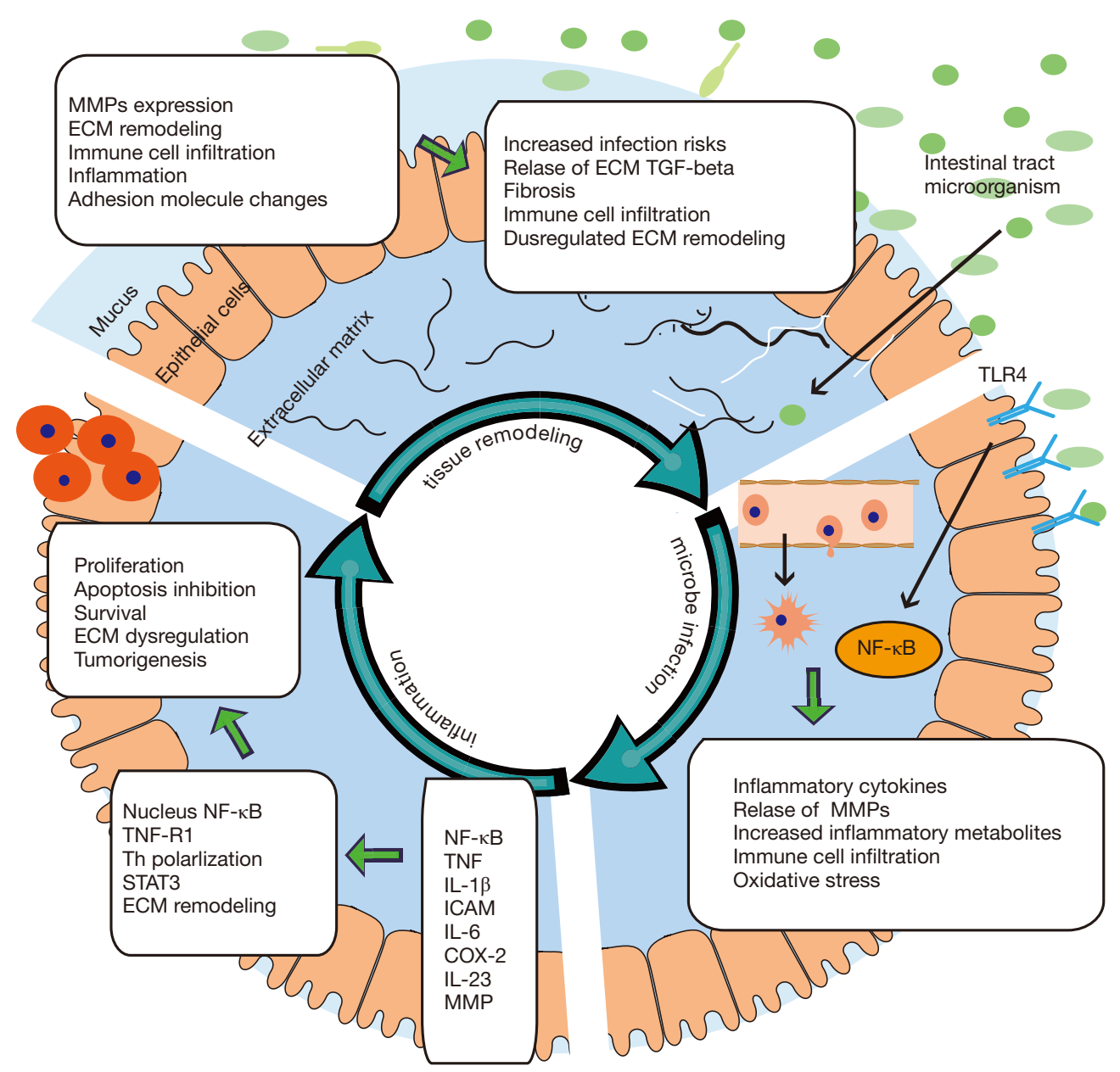

Figure 1 Risk factors for the pathology and inflammation of UC. NF- $\kappa B$, nuclear factor kappa B; TNF, tumor necrosis factor; IL, interleukin; MMPs, matrix metalloproteinases; ECM, extracellular matrix; COX-2, cyclooxygenase-2; TNF-R1, TNF receptor 1; ICAM, intercellular cell adhesion molecule.

activation of sustained inflammation. Then, inflammation and cytokines aggravate ECM remodeling and recruit immunity cells. Eventually, a mutually positive feedback of microbial invasion, inflammation, and ECM remodeling is established, which triggers continuous inflammation which may be responsible for the continuous inflammation in UC and related CRC (Figure 1).

Different subtypes of IBD, including UC and CD have many similarities or even overlaps in the location, pathological features, and treatment methods (1). The lesions of UC often appear in the epithelial layer of the colon only, progress in a spreading way, and are more likely to induce CRC. In contrast, CD spreads throughout the intestinal wall, the lesions are diffuse, and are more likely to spread to the small intestine side. Despite these distinctions, especially in inflammation activation, tissue remodeling, and gene mutation, the 2 diseases are very similar $(52,61)$. Therefore, the mechanism of inflammation in UC and related CRC summarized in this review possibly also aligns with CD. Similar to how controlling inflammation can reduce the risk of CRC, controlling inflammation may also help reduce CD-related cancers such as small bowel cancer.

\section{Acknowledgments}

Funding: None.

\section{Footnote}

Reporting Checklist: The authors have completed the 
Narrative Review reporting checklist. Available at https:// dx.doi.org/10.21037/apm-21-3138

Conflicts of Interest: All authors have completed the ICMJE uniform disclosure form (available at https://dx.doi. org/10.21037/apm-21-3138). The authors have no conflicts of interest to declare.

Ethical Statement: The authors are accountable for all aspects of the work in ensuring that questions related to the accuracy or integrity of any part of the work are appropriately investigated and resolved.

Open Access Statement: This is an Open Access article distributed in accordance with the Creative Commons Attribution-NonCommercial-NoDerivs 4.0 International License (CC BY-NC-ND 4.0), which permits the noncommercial replication and distribution of the article with the strict proviso that no changes or edits are made and the original work is properly cited (including links to both the formal publication through the relevant DOI and the license). See: https://creativecommons.org/licenses/by-nc-nd/4.0/.

\section{References}

1. D'Haens GRAM, Lindsay JO, Panaccione R, et al. Ulcerative Colitis: Shifting Sands. Drugs R D 2019;19:227-34.

2. Cui G, Yuan A. A Systematic Review of Epidemiology and Risk Factors Associated With Chinese Inflammatory Bowel Disease. Front Med (Lausanne) 2018;5:183.

3. Kobayashi T, Siegmund B, Le Berre C, et al. Ulcerative colitis. Nat Rev Dis Primers 2020;6:74.

4. Feuerstein JD, Isaacs KL, Schneider Y, et al. AGA Clinical Practice Guidelines on the Management of Moderate to Severe Ulcerative Colitis. Gastroenterology 2020;158:1450-61.

5. Ko CW, Singh S, Feuerstein JD, et al. AGA Clinical Practice Guidelines on the Management of Mildto-Moderate Ulcerative Colitis. Gastroenterology 2019;156:748-64.

6. Lopez A, Pouillon L, Beaugerie L, et al. Colorectal cancer prevention in patients with ulcerative colitis. Best Pract Res Clin Gastroenterol 2018;32-33:103-9.

7. Eaden JA, Abrams KR, Mayberry JF. The risk of colorectal cancer in ulcerative colitis: a meta-analysis. Gut 2001;48:526-35.

8. Yao D, Dong M, Dai C, et al. Inflammation and
Inflammatory Cytokine Contribute to the Initiation and Development of Ulcerative Colitis and Its Associated Cancer. Inflamm Bowel Dis 2019;25:1595-602.

9. Ohman L, Dahlén R, Isaksson S, et al. Serum IL-17A in newly diagnosed treatment-naive patients with ulcerative colitis reflects clinical disease severity and predicts the course of disease. Inflamm Bowel Dis 2013;19:2433-9.

10. Xie Z, Qu Y, Leng Y, et al. Human colon carcinogenesis is associated with increased interleukin-17-driven inflammatory responses. Drug Des Devel Ther 2015;9:1679-89.

11. Qi H, Yang H, Xu G, et al. Therapeutic efficacy of IL$17 \mathrm{~A}$ antibody injection in preventing the development of colitis associated carcinogenesis in mice. Immunobiology 2015;220:54-9.

12. Carrière J, Darfeuille-Michaud A, Nguyen HT. Infectious etiopathogenesis of Crohn's disease. World J Gastroenterol 2014;20:12102-17.

13. Farré R, Vicario M. Abnormal Barrier Function in Gastrointestinal Disorders. Handb Exp Pharmacol 2017;239:193-217.

14. Kleessen B, Kroesen AJ, Buhr HJ, et al. Mucosal and invading bacteria in patients with inflammatory bowel disease compared with controls. Scand J Gastroenterol 2002;37:1034-41.

15. Neurath MF. Targeting immune cell circuits and trafficking in inflammatory bowel disease. Nat Immunol 2019;20:970-9.

16. Barberis E, Joseph S, Amede E, et al. A new method for investigating microbiota-produced small molecules in adenomatous polyps. Anal Chim Acta 2021;1179:338841.

17. Arnold CN, Goel A, Blum HE, et al. Molecular pathogenesis of colorectal cancer: implications for molecular diagnosis. Cancer 2005;104:2035-47.

18. Netzer P, Forster C, Biral R, et al. Risk factor assessment of endoscopically removed malignant colorectal polyps. Gut 1998;43:669-74.

19. Kekilli M, Dagli U, Kalkan IH, et al. Low incidence of colorectal dysplasia and cancer among patients with ulcerative colitis: a Turkish referral centre study. Scand J Gastroenterol 2010;45:434-9.

20. Ashktorab H, Brim H, Hassan S, et al. Inflammatory polyps occur more frequently in inflammatory bowel disease than other colitis patients. BMC Gastroenterol 2020;20:170.

21. Laish I, Shurani A, Barkay O, et al. Low prevalence of dysplastic polyps in patients with ulcerative colitis. Clin Res Hepatol Gastroenterol 2017;41:204-9. 
22. Yang Y, Li L, Xu C, et al. Cross-talk between the gut microbiota and monocyte-like macrophages mediates an inflammatory response to promote colitis-associated tumourigenesis. Gut 2020;70:1495-506.

23. Franzosa EA, Sirota-Madi A, Avila-Pacheco J, et al. Gut microbiome structure and metabolic activity in inflammatory bowel disease. Nat Microbiol 2019;4:293-305.

24. Paramsothy S, Nielsen S, Kamm MA, et al. Specific Bacteria and Metabolites Associated With Response to Fecal Microbiota Transplantation in Patients With Ulcerative Colitis. Gastroenterology 2019;156:14401454.e2.

25. Tang Q, Cang S, Jiao J, et al. Integrated study of metabolomics and gut metabolic activity from ulcerative colitis to colorectal cancer: The combined action of disordered gut microbiota and linoleic acid metabolic pathway might fuel cancer. J Chromatogr A 2020;1629:461503.

26. Hirano T, Hirayama D, Wagatsuma K, et al. Immunological Mechanisms in Inflammation-Associated Colon Carcinogenesis. Int J Mol Sci 2020;21:3062.

27. Soleimani A, Rahmani F, Ferns GA, et al. Role of the NF$\kappa \mathrm{B}$ signaling pathway in the pathogenesis of colorectal cancer. Gene 2020;726:144132.

28. Greten FR, Eckmann L, Greten TF, et al. IKKbeta links inflammation and tumorigenesis in a mouse model of colitis-associated cancer. Cell 2004;118:285-96.

29. Baldwin AS. Control of oncogenesis and cancer therapy resistance by the transcription factor NF-kappaB. J Clin Invest 2001;107:241-6.

30. Seguella L, Rinaldi F, Marianecci C, et al. Pentamidine niosomes thwart $\mathrm{S} 100 \mathrm{~B}$ effects in human colon carcinoma biopsies favouring wtp53 rescue. J Cell Mol Med 2020;24:3053-63.

31. Ye X, Wu H, Sheng L, et al. Oncogenic potential of truncated RXR $\alpha$ during colitis-associated colorectal tumorigenesis by promoting IL-6-STAT3 signaling. Nat Commun 2019;10:1463.

32. Huang B, Chen Z, Geng L, et al. Mucosal Profiling of Pediatric-Onset Colitis and IBD Reveals Common Pathogenics and Therapeutic Pathways. Cell 2019;179:1160-1176.e24.

33. Popivanova BK, Kitamura K, Wu Y, et al. Blocking TNFalpha in mice reduces colorectal carcinogenesis associated with chronic colitis. J Clin Invest 2008;118:560-70.

34. Onizawa M, Nagaishi T, Kanai T, et al. Signaling pathway via TNF-alpha/NF-kappaB in intestinal epithelial cells may be directly involved in colitis-associated carcinogenesis. Am J Physiol Gastrointest Liver Physiol 2009;296:G850-9.

35. Rutgeerts P, Sandborn WJ, Feagan BG, et al. Infliximab for induction and maintenance therapy for ulcerative colitis. N Engl J Med 2005;353:2462-76.

36. Mizoguchi E, Mizoguchi A, Takedatsu H, et al. Role of tumor necrosis factor receptor 2 (TNFR2) in colonic epithelial hyperplasia and chronic intestinal inflammation in mice. Gastroenterology 2002;122:134-44.

37. Corredor J, Yan F, Shen CC, et al. Tumor necrosis factor regulates intestinal epithelial cell migration by receptordependent mechanisms. Am J Physiol Cell Physiol 2003;284:C953-61.

38. Craven B, Zaric V, Martin A, et al. Effect of genetic deletion or pharmacological antagonism of tumor necrosis factor alpha on colitis-associated carcinogenesis in mice. Inflamm Bowel Dis 2015;21:485-95.

39. Mitsuyama K, Sata M, Tanikawa K. Significance of interleukin-6 in patients with inflammatory bowel disease. Gastroenterol Jpn 1991;26:20-8.

40. Mitsuyama K, Sasaki E, Toyonaga A, et al. Colonic mucosal interleukin-6 in inflammatory bowel disease. Digestion 1991;50:104-11.

41. Chen YY, Ma ZB, Xu HY, et al. IL-6/STAT3/SOCS3 signaling pathway playing a regulatory role in ulcerative colitis carcinogenesis. Int J Clin Exp Med 2015;8:12009-17.

42. Grivennikov S, Karin E, Terzic J, et al. IL-6 and Stat3 are required for survival of intestinal epithelial cells and development of colitis-associated cancer. Cancer Cell 2009;15:103-13.

43. Chalaris A, Schmidt-Arras D, Yamamoto K, et al. Interleukin-6 trans-signaling and colonic cancer associated with inflammatory bowel disease. Dig Dis 2012;30:492-9.

44. Matsumoto S, Hara T, Mitsuyama K, et al. Essential roles of IL-6 trans-signaling in colonic epithelial cells, induced by the IL-6/soluble-IL-6 receptor derived from lamina propria macrophages, on the development of colitisassociated premalignant cancer in a murine model. J Immunol 2010;184:1543-51.

45. Friis S, Riis AH, Erichsen R, et al. Low-Dose Aspirin or Nonsteroidal Anti-inflammatory Drug Use and Colorectal Cancer Risk: A Population-Based, Case-Control Study. Ann Intern Med 2015;163:347-55.

46. Sheng H, Shao J, Morrow JD, et al. Modulation of apoptosis and Bcl-2 expression by prostaglandin E2 in human colon cancer cells. Cancer Res 1998;58:362-6.

47. Wang D, Wang H, Brown J, et al. CXCL1 induced by prostaglandin E2 promotes angiogenesis in colorectal 
cancer. J Exp Med 2006;203:941-51.

48. Sands BE, Sandborn WJ, Panaccione R, et al.

Ustekinumab as Induction and Maintenance Therapy for Ulcerative Colitis. N Engl J Med 2019;381:1201-14.

49. Neurath MF. IL-23 in inflammatory bowel diseases and colon cancer. Cytokine Growth Factor Rev 2019;45:1-8.

50. O'Connor PM, Lapointe TK, Beck PL, et al. Mechanisms by which inflammation may increase intestinal cancer risk in inflammatory bowel disease. Inflamm Bowel Dis 2010;16:1411-20.

51. Kirov S, Sasson A, Zhang C, et al. Degradation of the extracellular matrix is part of the pathology of ulcerative colitis. Mol Omics 2019;15:67-76.

52. Derkacz A, Olczyk P, Olczyk K, et al. The Role of Extracellular Matrix Components in Inflammatory Bowel Diseases. J Clin Med 2021;10:1122.

53. Koelink PJ, Overbeek SA, Braber S, et al. Collagen degradation and neutrophilic infiltration: a vicious circle in inflammatory bowel disease. Gut 2014;63:578-87.

54. Pedersen G, Saermark T, Kirkegaard T, et al. Spontaneous and cytokine induced expression and activity of matrix metalloproteinases in human colonic epithelium. Clin Exp Immunol 2009;155:257-65.

55. Bost F, Diarra-Mehrpour M, Martin JP. Inter-alphatrypsin inhibitor proteoglycan family--a group of proteins binding and stabilizing the extracellular matrix. Eur J

Cite this article as: Tang P, Zha L, Ye C, Zhou L. Research progress on the carcinogenesis mechanism of inflammation in ulcerative colitis: a narrative review. Ann Palliat Med 2021;10(11):11994-12002. doi: 10.21037/apm-21-3138
Biochem 1998;252:339-46.

56. Kessler S, Rho H, West G, et al. Hyaluronan (HA) deposition precedes and promotes leukocyte recruitment in intestinal inflammation. Clin Transl Sci 2008;1:57-61.

57. Gordon IO, Agrawal N, Goldblum JR, et al. Fibrosis in ulcerative colitis: mechanisms, features, and consequences of a neglected problem. Inflamm Bowel Dis 2014;20:2198-206.

58. Oshima H, Nakayama M, Han TS, et al. Suppressing TGF $\beta$ signaling in regenerating epithelia in an inflammatory microenvironment is sufficient to cause invasive intestinal cancer. Cancer Res 2015;75:766-76.

59. Walter L, Canup B, Pujada A, et al. Matrix metalloproteinase 9 (MMP9) limits reactive oxygen species (ROS) accumulation and DNA damage in colitisassociated cancer. Cell Death Dis 2020;11:767.

60. Zhou G, Wu H, Lin J, et al. TRIM21 Is Decreased in Colitis-associated Cancer and Negatively Regulates Epithelial Carcinogenesis. Inflamm Bowel Dis 2021;27:458-68.

61. Yu YR, Rodriguez JR. Clinical presentation of Crohn's, ulcerative colitis, and indeterminate colitis: Symptoms, extraintestinal manifestations, and disease phenotypes. Semin Pediatr Surg 2017;26:349-55.

(English Language Editor: J. Jones) 